\title{
Correlation of omega-3 levels in serum phospholipid from 2053 human blood samples with key fatty acid ratios Bruce J Holub*1, Mike Wlodek ${ }^{2}$, William Rowe ${ }^{2}$ and Jerry Piekarski ${ }^{3}$
}

Address: ${ }^{1}$ Department of Human Health and Nutritional Sciences, University of Guelph, Guelph, Ontario, N1G 2W1, Canada, ${ }^{2}$ Nutrasource Diagnostics Inc. 120 Research Lane, Suite 203 University of Guelph Research Park Guelph, Ontario, N1G 0B4, Canada and ${ }^{3}$ Lipid Analytical Laboratories Inc. 150 Research Lane, Room 100 University of Guelph Research Park Guelph, Ontario, N1G 4T4, Canada

Email: Bruce J Holub* - bholub@uoguelph.ca; Mike Wlodek - mwlodek@nutrasource.ca; William Rowe - wrowe@nutrasource.ca; Jerry Piekarski - lal@idirect.ca

* Corresponding author

Published: 24 December 2009

Nutrition Journal 2009, 8:58 doi:10.1/86/1475-289/-8-58

This article is available from: http://www.nutritionj.com/content/8/I/58

(c) 2009 Holub et al; licensee BioMed Central Ltd.

This is an Open Access article distributed under the terms of the Creative Commons Attribution License (http://creativecommons.org/licenses/by/2.0), which permits unrestricted use, distribution, and reproduction in any medium, provided the original work is properly cited.

\begin{abstract}
Background: This research was conducted to explore the relationships between the levels of omega-3 fatty acids in serum phospholipid and key fatty acid ratios including potential cut-offs for risk factor assessment with respect to coronary heart disease and fatal ischemic heart disease.
\end{abstract}

Methods: Blood samples $(n=2053)$ were obtained from free-living subjects in North America and processed for determining the levels of total fatty acids in serum phospholipid as omega-3 fatty acids including EPA (eicosapentaenoic acid, 20:5 n-3) and DHA (docosahexaenoic acid, 22:6n-3) by combined thin-layer and gas-liquid chromatographic analyses. The omega- 3 levels were correlated with selected omega-6: omega-3 ratios including AA (arachidonic acid, 20:4n-6): EPA and AA:(EPA+DHA). Based on previously-published levels of omega- 3 fatty acids considered to be in a 'lower risk' category for heart disease and related fatality, 'lower risk' categories for selected fatty acid ratios were estimated.

Results: Strong inverse correlations between the summed total of omega-3 fatty acids in serum phospholipid and all four ratios (omega-6:omega-3 (n-6:n-3), AA:EPA, AA:DHA, and $A A:(E P A+D H A))$ were found with the most potent correlation being with the omega-6:omega-3 ratio $\left(R^{2}=0.96\right)$. The strongest inverse relation for the EPA+DHA levels in serum phospholipid was found with the omega-6: omega-3 ratio $\left(R^{2}=0.94\right)$ followed closely by the AA: $(E P A+D H A)$ ratio at $\mathrm{R}^{2}=0.88$. It was estimated that $95 \%$ of the subjects would be in the 'lower risk' category for coronary heart disease (based on total omega- $3 \geq 7.2 \%$ ) with omega-6:omega- 3 ratios $<4.5$ and AA:(EPA+DHA) ratios $<1.4$. The corresponding ratio cut-offs for a 'lower risk' category for fatal ischemic heart disease $(E P A+D H A \geq 4.6 \%)$ were estimated at $<5.8$ and $<2$. , respectively.

Conclusions: Strong inverse correlations between the levels of omega- 3 fatty acids in serum (or plasma) phospholipid and omega-6: omega-3 ratios are apparent based on this large database of 2053 samples. Certain fatty acid ratios may aid in cardiovascular disease-related risk assessment if/ when complete profiles are not available. 


\section{Introduction}

The fatty acid composition of serum (or plasma) phospholipid has become established as a valid biochemical marker for assessing the physiological status of various fatty acids including predictive correlations with the dietary intakes of fish-derived omega-3 fatty acids including EPA (eicosapentaenoic acid, 20:5 n-3) and DHA (docosahexaenoic acid, 22:6 n-3) [1,2]. Population studies have shown an inverse relation between total omega-3 fatty acids in blood serum phospholipid and the risk for coronary heart disease with percentages of total omega- $3 \geq 7.2$ being associated with a 31\% lower risk [3,4]. Furthermore, DHA levels (as percent of total fatty acids in serum phospholipid) of $\geq 4.5$ have been associated with a $34 \%$ lower risk for coronary heart disease $[3,4]$. With respect to the risk of fatal ischemic heart disease, EPA+DHA (summed) levels amounting to at least $4.6 \%$ of total fatty acids in the serum phospholipid were associated with a $70 \%$ lower risk as compared to those with much lower levels of these fatty acids $[4,5]$.

Since the omega- 6 fatty acid, AA (arachidonic acid, 20:4 n6 ), found in abundance in various cells and tissues including serum phospholipid can be readily converted into pro-inflammatory eicosanoids and other products associated with inflammatory processes and chronic disorders in contrast to EPA [6], the AA:EPA ratio in serum phospholipid has been studied in relation to the risk of chronic disorders. These studies have, as an example, indicated that the AA/EPA ratio in serum (or plasma) phospholipid correlates positively with clinical symptoms of depression $[7,8]$; furthermore, higher ratios of AA:DHA were associated with greater neuroticism [8]. Others have implicated the abundance of the summed omega- 6 relative to the omega-3 fatty acids in human plasma phospholipid with respect to chronic disorders [9]. While the percentages of omega-3 fatty acids or key ratios (e.g., AA:EPA ratio) in serum (or plasma) phospholipid and disease risk are often given in published papers, the percentages and various key fatty acid ratios are usually not provided simultaneously.

In view of the availability of an extremely large database (over 2000 samples) of subjects from North America with complete fatty acid profiles of their serum phospholipid, the following were of interest: 1) to determine the relationship between the percentage of total fatty acids in serum phospholipid as total omega-3, DHA, and (EPA+ DHA)-risk factors for coronary heart disease and fatal ischemic heart disease (plus other chronic disorders) to the various ratios (omega-6:omega-3, AA:EPA, AA:DHA, and $\mathrm{AA}:(\mathrm{EPA}+\mathrm{DHA}) ; 2)$ to compare the relative strengths of these correlations (fatty acid percentages with ratios); 3 ) to determine the corresponding cut-points (95 percentile) for the (omega-6:omega-3), AA:EPA, AA:DHA, and
$\mathrm{AA}(\mathrm{EPA}+\mathrm{DHA})$ ratios which are associated with a $95 \%$ likelihood of the percentage of total fatty acids in serum phospholipid as omega-3, DHA, and EPA+DHA being in the aforementioned 'lower-risk' category [3-5].

\section{Methods \\ Study Population and Blood Samples}

Blood samples $(\mathrm{n}=2053)$ were obtained from free-living subjects (both genders) from across Canada and the United States who initiated contact with their health professional or health care provider to request analyses of their serum phospholipid for fatty acid profiling and cardiovascular risk estimations based on the relations between the levels of long-chain omega-3 fatty acids for coronary heart disease and fatal ischemic heart disease [3$5]$. The wide diversity in the diets (including fish intakes) and use of supplements or functional foods containing $\mathrm{DHA}+\mathrm{EPA}$ in this free-living population provided a convenient spread of blood levels of omega- 3 fatty acids and fatty acid ratios. The receiving and processing of these samples were approved by the Human Ethics Committee of the University of Guelph. Since the primary goal of this study was to evaluate the relationship between the levels (percentages) of total omega-3 fatty acids, DHA, and (EPA+DHA) in the biomarker (serum phospholipid) and specific fatty acid ratios (omega-6:omega-3, AA/EPA, AA/ $\mathrm{DHA}$, and $\mathrm{AA} /(\mathrm{EPA}+\mathrm{DHA}))$, no restriction was placed on the subjects (including blood sampling being permitted in the fasted or postprandial state). Following collection of the blood samples by venipuncture into a Vacutainer tube, the serum was collected for processing and analyses via centrifugation $[10,11]$.

\section{Fatty Acid Analyses of Serum Phospholipid}

Fatty acid compositions of total serum phospholipid were determined based on previous studies [10,11]. Lipids were extracted from the serum samples according to the method of Folch et al.[12] and the serum phospholipids were separated from the neutral lipids by thin-layer chromatography $[10,11]$. The fatty acids methyl esters were prepared from the isolated phospholipid fraction by the method of Morrison and Smith [13] and were analyzed on a Varian 3400 gas-liquid chromatograph (Palo Alto, CA) with a 60-metre DB-23 capillary column $(0.32 \mathrm{~mm}$ internal diameter).

\section{Statistical Analysis}

The database was assessed for completeness and it was determined that a total of 2053 blood test analyses contained results for each of the bio-markers which were to be analyzed. The database was formatted in a Microsoft Excel $^{\mathrm{TM}}$ (EXCEL) spreadsheet. A preliminary analysis using the EXCEL correlation coefficient function and trend-line analysis graphing function was performed on all of the data. Upon review of the resulting correlation coefficients 
for all comparisons, it was determined that statistically significant correlations could be ascertained for the dependent variables (x-axis) as Total Omega-3, DHA, and (EPA+DHA).

Independent variables (y-axis) for "yTotal Omega-6/Total Omega-3", "AA/EPA", "AA/DHA", and "AA/(EPA+DHA)" ratios were selected. Data for EPA, DHA, total Omega-3, total Omega-6, and AA were extracted from the original spreadsheet into a new spreadsheet for analytical purposes. The EPA and DHA levels were summed to calculate "EPA+DHA" values for each of the samples. Calculations were made for the "Total Omega-6/Total Omega-3", "AA/ EPA", "AA/DHA", and "AA/(EPA+DHA)" ratio values for each sample. Calculations using the correlation function were made using linear, polynomial, $\log$, exponential and power curve fits. In every case, the power curve provided the highest correlation coefficient and Power was therefore selected to model the data. A curve based on the Power function was plotted and the $\mathrm{R}^{2}$ was recorded. This curve is used for graphical purposes only. The $\mathrm{R}^{2}$ was used to compare between the different Y-values (i.e. AA/EPA ratio, n-6/n3 ratio, etc) so that a determination could be made as to which ratio "fit" the data best. The Power Function was not used in determining the cut-offs, these cutoffs are based on distribution calculations as described below [14].

The ninety-five percent (95\%) confidence intervals [15] for the new independent variable cut-offs (selected fatty acid ratios) were established by analyzing the data which was above the known cut-off values for each dependent variable (fatty acid components as $\%$ of total fatty acids in serum phospholipid ) and their associated 'lower risk' status.

Calculations were validated by the University of Guelph's Department of Mathematics and Statistics.

\section{Results}

The fatty acid data available from our very large sample (n $=2053$ subjects) on the levels of total omega-3, DHA, and $\mathrm{EPA}+\mathrm{DHA}$ as percentages of total fatty acids in serum phospholipid is considered one of the largest ever processed. Based on the estimated 'lower risk' categories for the risk of cardiovascular disease-related outcomes [3-5], the proportion of our population considered to be at potential 'risk' could be determined. Based on a 'risk' cutoff' of $\geq 7.2 \%$ for total omega- 3 fatty acids and $\geq 4.5 \%$ for DHA $[3,4]$ as \% of fatty acids in serum phospholipid, $49.5 \%$ (1017 of 2053) and 53.9\% (1106 of 2053) of our samples would be placed in the 'risk' category. In the case of EPA+DHA levels, where \% values of $\geq 4.6$ appears associated with a considerably lower risk of fatal ischemic heart disease [4,5], 34.0\% (699 of 2053) could be classified in the 'risk' category.

Statistical analysis revealed very strong and significant inverse correlations between the sum of total omega-3 fatty acids in serum phospholipid and all four ratios (Figure 1) with the most potent correlation $\left(\mathrm{R}^{2}=0.96\right)$ being with the omega- 6 fatty acids (sum): omega-3 fatty acids (sum) ratio followed (in order of diminishing potency) by the ratios of AA:(EPA+DHA), AA:EPA, and AA:DHA.

In the case of $\%$ of fatty acids as DHA in serum phospholipid (Figure 2), the most potent inverse correlation was found with the AA:DHA ratio $\left(\mathrm{R}^{2}=0.81\right)$ and the weakest correlation was with the AA:EPA ratio $\left(\mathrm{R}^{2}=0.46\right)$. The other two correlations with the omega-6:omega-3 ratio and the AA: (EPA+DHA) fell between these extremes with $\mathrm{R}^{2}$ values of 0.74 and 0.71 , respectively.

Correlational analyses for the \% of total fatty acids in serum phospholipid (Figure 3 ) as EPA+DHA showed very strong inverse relations with all four ratios. The strongest relation was found with the omega- 6 :omega- 3 ratio $\left(R^{2}=\right.$ $0.94)$ followed closely by the AA: $(E P A+D H A)$ ratio $\left(R^{2}=\right.$ $0.88)$ and then the AA:DHA $\left(\mathrm{R}^{2}=0.79\right)$ and AA:EPA $\left(\mathrm{R}^{2}=\right.$ 0.77 ) ratios.

Table 1 gives the cut-off values for the four fatty acid ratios which would place $95 \%$ of the fatty acid levels in the 'low risk' category for coronary heart disease (total omega- $3 \geq$ 7.2 and DHA $\geq 4.5 \%$ of total fatty acids in serum phospholipid) and fatal ischemic heart disease (sum $\mathrm{EPA}+\mathrm{DHA} \geq 4.6 \%$ ).

For each of the latter three parameters, the lowest numerical cut-off ratios were for AA:(EPA+DHA) showing cutoffs of $<1.4,<0.45$, and $<2.1$, respectively. The highest numerical cut-off ratios for total omega-3, DHA, and EPA+DHA were AA:EPA (<5.0), omega-6:omega-3 (<3.0), and AA:EPA $(<9.2)$, respectively.

\section{Discussion}

The present results indicate that very strong and highly significant inverse relationships exist for the levels of total omega-3 fatty acids, DHA, and EPA+DHA (sum) as a \% of total fatty acids in serum phospholipid (Figures 1,2 and 3 ). These results are of interest since the levels of these fatty acids have been correlated with the risk for coronary heart disease and fatal ischemic heart disease [3-5]. Furthermore, various groups have reported on either these fatty acid levels or ratios such as the omega-6:omega-3, AA:EPA, and EPA:DHA ratios but not both simultaneously in chronic disease risk assessment [3-5,7-9]. Our present study provides some guidance as to what appropriate ratio cut-offs may be considered for cardiovascular 


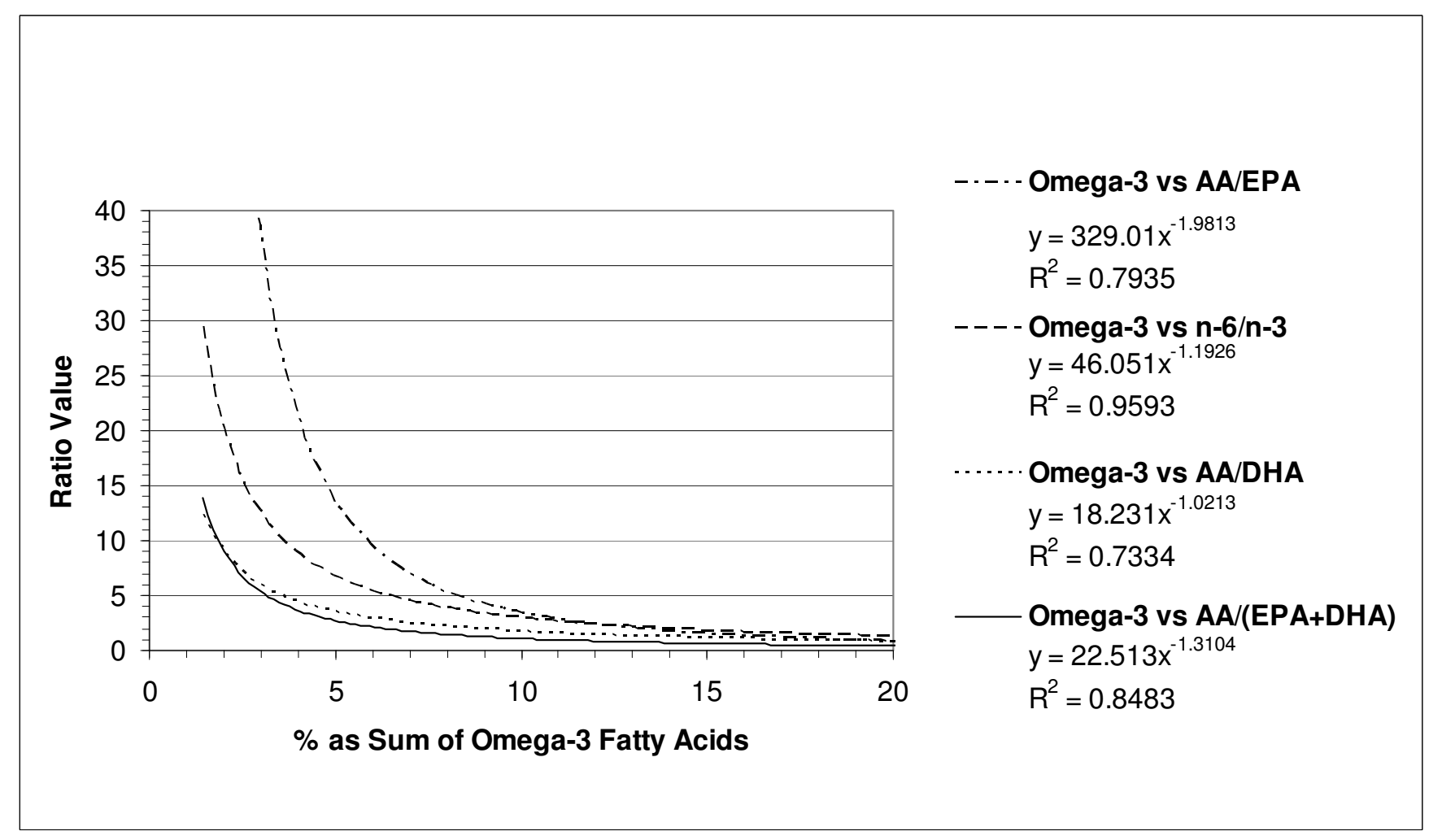

Figure I

Relationships between \% of Total Fatty Acids in Serum Phospholipid as Omega-3 and Fatty Acid Ratios.

disease risk assessment (Table 1). Interestingly, the most potent relationships (inverse) for any fatty acid parameter and ratio was for total omega-3 fatty acids and the omega6:omega-3 ratio $\left(R^{2}=0.96\right)$. In this case, a ratio of $<4.5$ would place $95 \%$ of the subjects in a 'lower risk' category for coronary heart disease based on an omega-3 (summed) risk cut-off of $\geq 7.2 \%[3,4]$. As might be expected, the AA:DHA ratio exhibited the strongest inverse relation to the DHA levels. The omega-6:omega-3 ratio also showed the strongest inverse relation to the EPA+DHA levels in serum phospholipid $\left(R^{2}=0.94\right)$ and a cut-off ratio of $<5.8$ would be expected to place $95 \%$ of the subjects in a 'lower risk' category for fatal ischemic heart disease based on $\geq 4.6 \%$ of the total fatty acids being present as EPA+DHA [4,5].

The major fatty acids contributing to the total omega-3 fatty acids in human serum phospholipid are EPA+DHA along with docosapentaenoic acid with alpha-linolenic acid being a very minor component despite its abundance in dietary plant oils with AA plus LA (linoleic acid, 18:2 n6 ) being the major contributors to the total omega- 6 fatty acids $[16,17]$. Based on typical baseline fatty acid ratios [16] in serum phospholipid for groups of North American males (omega-6: omega-3 = 8.1, AA:EPA = 16.2, AA:DHA
$=4.2$, and $\mathrm{AA}:(\mathrm{EPA}+\mathrm{DHA})=3.3))$, none of these average ratios fall in the lower risk categories as defined in Table 1. Japanese males [17] with much lower corresponding ratios of 2.3, 1.7, 0.9, and 0.6 , respectively, would fall in the 'lower risk' categories for coronary heart disease based on total omega-3 $(\geq 7.2)$ and fatal ischemic heart disease $(\mathrm{EPA}+\mathrm{DHA} \geq 4.6)$ based on Table 1 . Their chronic daily intake of EPA+DHA, mainly from fish/seafood, was estimated to average $1500 \mathrm{mg} /$ day [17]. Interestingly, daily supplementation of North American males with $630 \mathrm{mg}$ EPA plus $640 \mathrm{mg}$ DHA for 21 days lowered their ratios into or towards the 'lower risk' categories with newlymodified ratios of 3.8, 5.1, 2.1, and 1.5, respectively [16].

It is of interest to note that the omega- 3 index, a measure of the $\%$ of total fatty acids in the red blood cell represented by the sum of EPA + DHA, has been suggested as a risk factor for death from coronary heart disease [18]. Also, a very strong correlation between the omega- 3 index and the $\%$ of total fatty acids in plasma phospholipid as EPA+DHA $(r=0.91)$ was reported [18]. The cut-off value referred to herein (EPA+DHA in serum/plasma phospholipid) based on the literature for a reduced risk of fatal ischemic heart disease $[4,5]$ of $\geq 4.6 \%$ can be compared to $\geq 8.0 \%$ for the omega-3 index [18]. Interestingly, the 


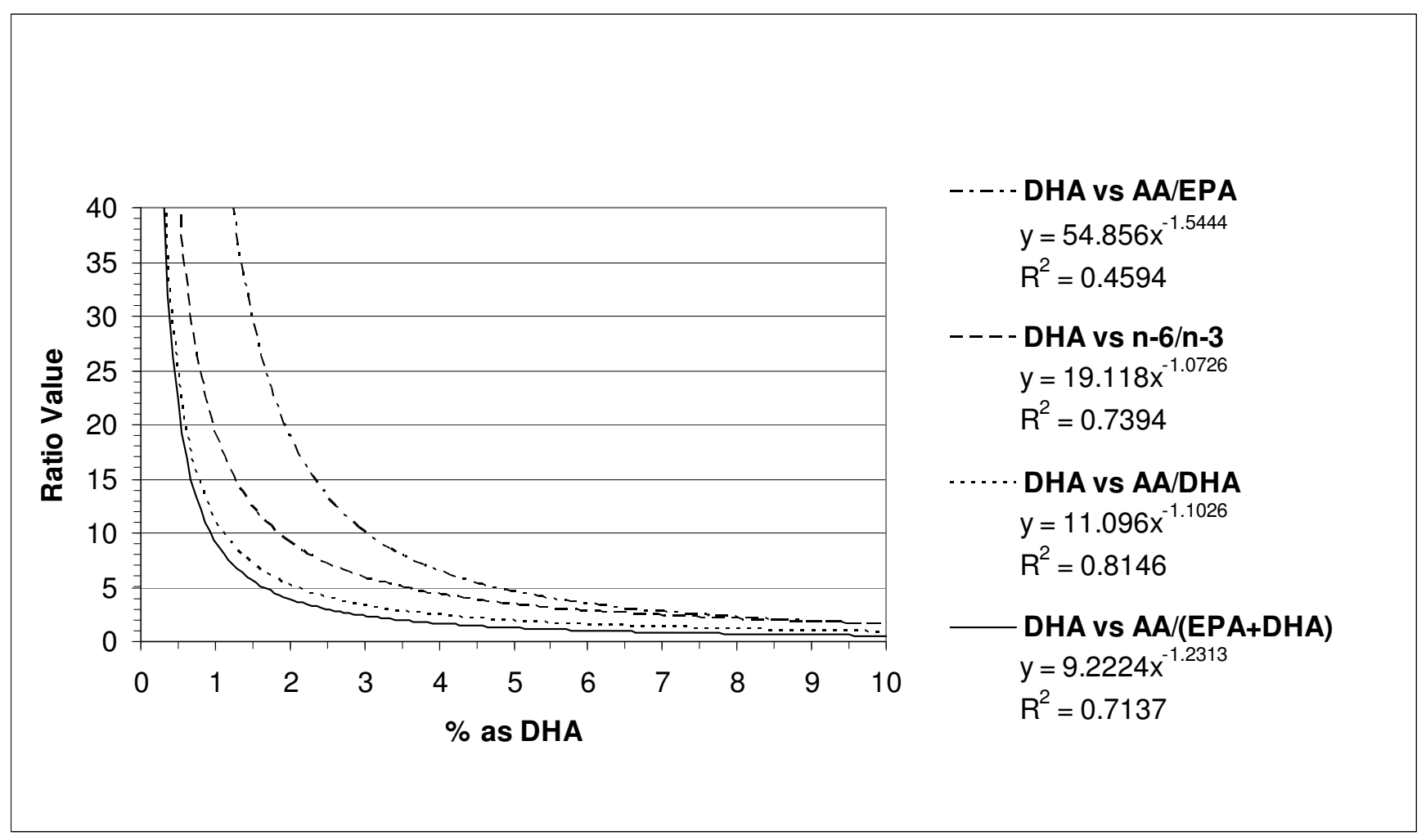

Figure 2

Relationships between \% of Total Fatty Acids in Serum Phospholipid as DHA and Fatty Acid Ratios.

$\geq 8.0 \%$ level gives a value of $\geq 4.7 \%$ for $\mathrm{EPA}+\mathrm{DHA}$ if the former is extrapolated to plasma phospholipid based on the correlation equation provided [18].

It needs to be emphasized that the relations between the fatty acid levels and the ratios presented herein are considered independent of the health or clinical condition of interest. Specific information obtained on the majority $(70 \%)$ of the subjects in our population providing blood samples (numbering 2053) indicated that 54\% were males and $46 \%$ were females. Of these, the average age for both genders was 54.7 years with the average for males and females being 55.2 and 54.2 years, respectively.

The estimated 'risk' fatty acid ratios and cut-offs as presented herein were directed to cardiovascular disease and fatal ischemic heart disease and could be of value if/when complete profiles are not available. Thus, they would not, of course, apply to depression, neuroticism, and other chronic conditions where ratios have been of interest $[7,8]$. It is also emphasized that the biomarker for the present study is serum phospholipid such that plasma phospholipid but not whole blood or red blood cell data could be substituted.

\section{Conclusions}

In summary, our study showed robust inverse curvilinear relationships between the summed omega- 3 fatty acids in serum phospholipid (biomarker) as well as the (EPA+DHA) levels and the omega-6/omega-3 and the AA/ (EPA+DHA) ratios based on fatty acid analyses of 2053 human blood samples. Omega-6/omega-3 ratios $<4.5$ were estimated to support a 'lower risk' category with $95 \%$ confidence for the risk of coronary heart disease while AA/ (EPA+DHA) ratios <2.1 would be expected to provide a corresponding lower risk category for fatal ischemic heart disease.

\section{List of Abbreviations}

AA: Arachidonic acid, 20:4 n-6; DHA: Docosahexaenoic acid, 22:6 n-3; EPA: Eicosapentaenoic acid, 20:5 n-3; LA: Linoleic acid, 18:2 n-6

\section{Competing interests}

B.J. Holub, M. Wlodek, and W. Rowe all hold ownership shares in Nutrasource Diagnostics Inc. This company provides omega-3 fatty acid blood testing services for health care groups and their clients in North America. J. Piekarski holds an ownership share in Lipid Analytical Laboratories Inc. 


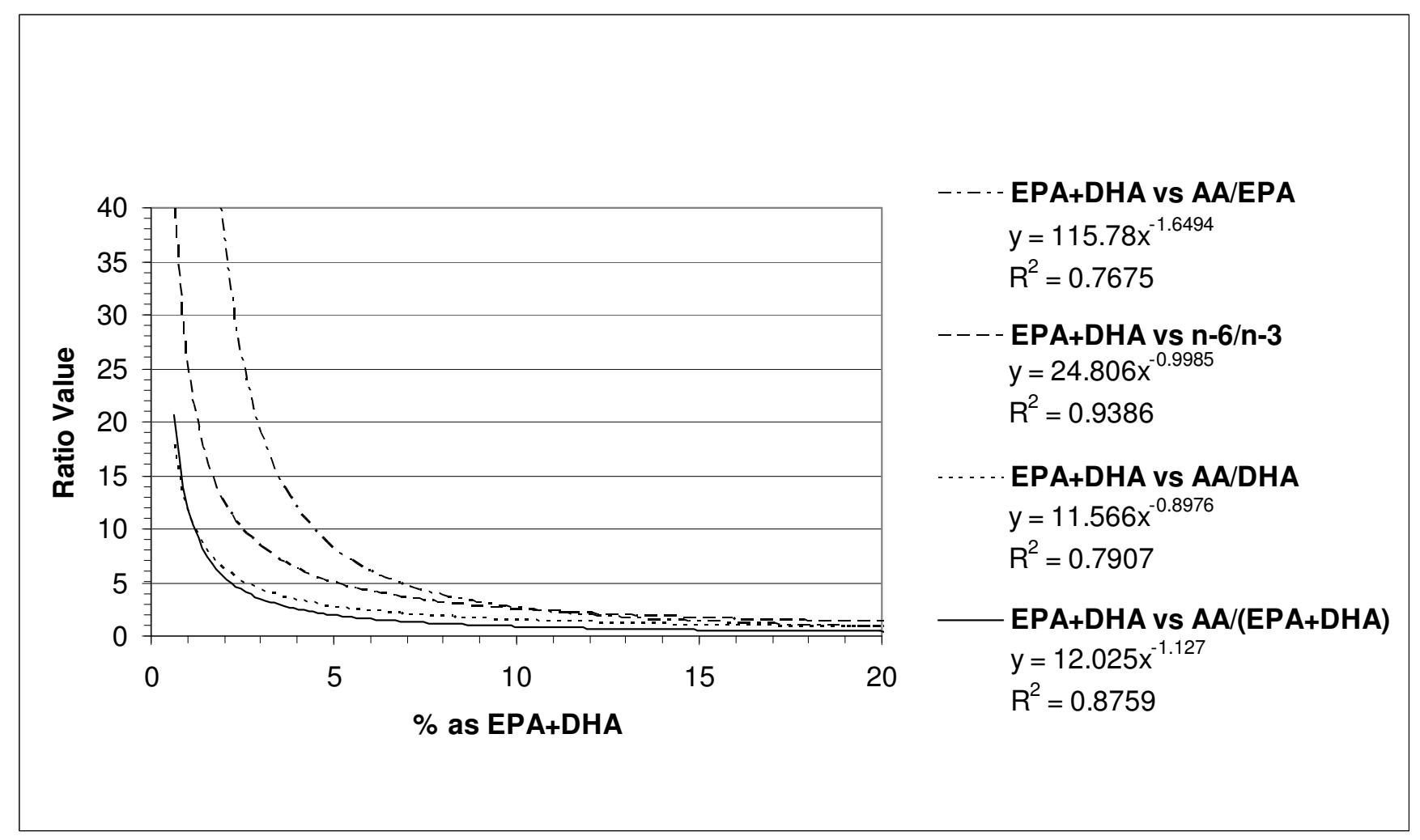

\section{Figure 3}

Relationships between \% of Total Fatty Acids in Serum Phospholipid as EPA+DHA and Fatty Acids Ratios.

Table I: Cut-off Values for the Fatty Acid Ratios which place the Levels of Total Omega-3 Fatty Acids, DHA, and EPA+DHA in the 'Lower Risk' Categories with 95\% Confidence Level

Cut-offs for Fatty Acid Ratios

Fatty Acid Levels * (Lower Risk Category)

\begin{tabular}{cccc}
\hline Omega-6:Omega-3 & AA:EPA & AA:DHA & AA:(EPA+DHA) \\
\hline$<4.5$ & $<5.0$ & $<1.8$ & $<1.4$ \\
$<3.0$ & $<1.1$ & $<1.5$ & $<0.45$ \\
$<5.8$ & $<9.2$ & $<2.5$ & $<2.1$
\end{tabular}

Total Omega-3 $(\geq 7.2)$

$\operatorname{DHA}(\geq 4.5)$

$\mathrm{EPA}+\mathrm{DHA}(\geq 4.6)$

$<5.8$

$<9.2$

$<2$. 1

* The 'lower risk' categories for total omega-3, DHA, and EPA+DHA levels (as \% of total fatty acids in serum phospholipid) are for coronary heart disease in the case of total omega-3 and DHA $[3,4]$ and for fatal ischemic heart disease in the case of EPA+DHA [4,5]. 


\section{Authors' contributions}

$\mathrm{BJH}$ was responsible for the writing of this manuscript including interpretation of the data. MW contributed to the statistical analyses of the data with advice from the Department of Mathematics and Statistics, University of Guelph. WR served as project coordinator. JP performed all the serum phospholipid fatty acid analyses on the blood samples. All authors have read and approved the final manuscript.

\section{References}

I. Hjartaker A, Lund E, Bjerve KS: Serum phospholipid fatty acid composition and habitual intake of marine foods registered by a semi-quantitative food frequency questionnaire. Eur J Clin Nutr 1997, 5 I (I I):736-742.

2. Kobayashi M, Sasaki S, Kawabata T, Hasegawa K, Akabane M, Tsugane A: Single measurement of serum phospholipid fatty acid as a biomarker of specific fatty acid intake in middle-aged Japanese men. Eur J Clin Nutr 200I, 55:643-650.

3. Simon JA, Hodgkins ML, Browner WS, Neuhaus JM, Bernert JT, Hulley SB: Serum fatty acids and the risk of coronary heart disease. Am J Epidemiol 1995, 142:469-476.

4. Holub DJ, Holub BJ: Omega-3 fatty acids from fish oils and cardiovascular disease. Mol Cell Biochem 2004, 263:217-225.

5. Lemaitre RN, King IB, Mozaffarian D, Kuller LH, Tracy RP, Siscovick DS: $\mathbf{n - 3}$ Polyunsaturated fatty acids, fatal ischemic heart disease, and nonfatal myocardial infarction in older adults: The Cardiovascular Heart Study. Am J Clin Nutr 2003, 77:319-325.

6. Das UN: Can essential fatty acids reduce the burden of disease(s)? Lipids Health Dis 2008, 7:9.

7. Adams PB, Lawson S, Sangiorski A, Sinclair AJ: Arachidonic acid to eicosapentaenoic acid ratio in blood correlates positively with clinical symptoms of depression. Lipids 1996, 3 I (Suppl):SI57-SI6I.

8. Conklin SM, Manuck SB, Yao JK, Flory JD, Hibbeln JR, Muldoon MF: High $\omega-6$ and low $\omega-3$ fatty acids are associated with depressive symptoms and neuroticism. Psychosom Med 2007, 69:932-934.

9. Lands WE, Libelt B, Morris A, Kramer NC, Prewitt TE, Bowen P, Schmeisser D, Davidson MH, Burns J: Maintenance of lower portions of (n-6) eicosanoid precursors in phospholipids of human plasma in response to added dietary $(n-3)$ fatty acids. Biochim Biophys Acta 1992, I I80(2): 147-162.

10. Dewailly EE, Blanchet C, Gingras S, Lemieux S, Sauve L, Bergeron J, Holub BJ: Relations between $\mathbf{n - 3}$ fatty acid status and cardiovascular disease risk factors among Quebecers. Am J Clin Nutr 200I, 74(5):603-6II.

II. Stark KD, Holub B]: Differential eicosapentaenoic acid elevations and altered cardiovascular disease risk factor responses after supplementation with docosahexaenoic acid in postmenopausal women receiving and not receiving hormone replacement therapy. Am J Clin Nutr 2004, 79(5):765-773.

12. Folch J, Lees M, Sloane-Stanley GH: A simple method for the isolation and purification of total lipids from animal tissues. I Biol Chem 1957, 226(I):497-509.

13. Morrison WR, Smith LM: Preparation of fatty acid methyl esters and dimethylacetals from lipids with boron fluoride methanol. J Lipid Res 1964, 5:600-608.

14. Draper N, Smith H: Applied Regression Analysis. 3rd edition. Wiley Publishing; 1998.

15. Fleiss JL, Levin B, Paik MC: Statistical methods for rates and proportions (Third Addition). Wiley Series in Probability and statistics 2004 [http://www3.interscience.wiley.com/cgi-bin/bookhome/ $106599353 />]$.

16. Rose EL, Holub B]: Effects of a liquid egg product containing fish oil on selected cardiovascular disease risk factors: $A$ randomized crossover trial. Food Res Intern 2006, 39:910-916.

17. Kobayashi M, Sasaki S, Kawabata T, Hasegawa K, Akabane M, Tsugane $\mathrm{S}$ : Single measurement of serum phospholipid fatty acid as a biomarker of specific fatty acid intake in middle-aged Japanese men. Eur J Clin Nutr 200I, 55:643-650.
18. Harris WS, von Schacky C: The Omega-3 Index: a new risk factor for death from coronary heart disease? Prev Med 2004, 39:212-220.
Publish with Bio Med Central and every scientist can read your work free of charge

"BioMed Central will be the most significant development for disseminating the results of biomedical research in our lifetime. "

Sir Paul Nurse, Cancer Research UK

Your research papers will be:

- available free of charge to the entire biomedical community

- peer reviewed and published immediately upon acceptance

- cited in PubMed and archived on PubMed Central

- yours - you keep the copyright

Submit your manuscript here:

http://www.biomedcentral.com/info/publishing_adv.asp
BioMedcentral 